REDES- Revista hispana para el análisis de redes sociales

Vol.21,\#1, Diciembre 2011

http://revista-redes.rediris.es

\title{
Introducción: ¿en qué punto se encuentra el análisis de redes en Historia?
}

\author{
Michel Bertrand - Université de Toulouse-Instituto Universitario de Francia \\ Sandro GUZZI-HEEB - Universidad de Lausanne \\ Claire Lemercier - Centro de sociología de las organizaciones (UMR 7116 \\ CNRS-Sciences Po $)^{1}$
}

\begin{abstract}
Resumen
El concepto de red está ampliamente aceptado en el vocabulario de las ciencias sociales. En historia, la introducción del vocabulario de las redes ha estado ligado frecuentemente a aproximaciones situadas en una escala « micro » y que trataban de subrayar la agencia individual. Desde los años 1990, un análisis de redes más formalizado ha aparecido de forma episódica, y desigual según las áreas lingüísticas, en otros trabajos históricos fundados, por el contrario, en observaciones sistemáticas a una escala macro. Después de 30 años de una integración de la categoría en el campo de la historia, de los cuestionamientos con los que está asociada y de las metodologías que implica, emerge un verdadero "savoir-faire" del historiador tal y como lo muestran las investigaciones que movilizan efectivamente los análisis de redes. Sin embargo si éstos han demostrado ampliamente su interés en algunas áreas específicas de la historia, el diálogo entre aquellos que la practican es escaso. Nuestro deséo, mostrando las relaciones y las differencias que existen entre textos provenientes de países y sub-disciplinas variadas de la disciplina, es promover dicho diálogo.
\end{abstract}

Palabras clave: Historia - Redes sociales.

\begin{abstract}
The concept of network has largely been adopted in the vocabulary of social sciences. Historical research using the vocabulary of networks has often been associated to a "micro" approach aiming to highlight individual agency. Since 1990 a more formalized approach to network analysis has unevenly appeared, depending on the linguistic area, in other historical research based, on the contrary, on systematic macro scale observations. After 30 years of integrating the questions and the methods of the network approach into historical research it is possible to observe the emergence of a true "savoir faire" of historians as current historical research mobilizing the network approach can demonstrate. However, although the interest of applying a network approach in certain specific domains has been largely shown, there is still little dialog among researchers who practice it. By showing the relations and the differences between texts coming from different countries and diversified sub-disciplines we wish to contribute to such dialogue.
\end{abstract}

Key words: History - Social networks.

\footnotetext{
${ }^{1}$ Enviar correspondencia a: Claire Lemercier claire.lemercier@sciences-po.org
} 
REDES- Revista hispana para el análisis de redes sociales

Vol.21,\#1, Diciembre 2011

http://revista-redes.rediris.es

El concepto de red está ampliamente aceptado en el vocabulario de las ciencias sociales. Si en un principio, sobretodo en sociología, estuvo ligado al cuestionamiento de categorías estructurales como la de clase, contribuyó ampliamente al renacer del interés por los llamados « espacios informales », las relaciones personales, o incluso la capacidad de los individuos de construir, al menos parcialmente, su propio entorno. De este modo, la introducción del vocabulario de las redes ha estado ligado frecuentemente a aproximaciones situadas en una escala « micro » y que trataban de subrayar la agencia individual, tanto si se reivindicaban o no de la micro-historia italiana o francesa. Si estas corrientes habían insistido particularmente en los conceptos de configuraciones de relaciones 0 , por ejemplo, de «frentes de parentesco», y si sus promotores conocían diferentes investigaciones de la sociología de las redes sociales, no habían, en general, escogido utilizar las formalizaciones gráficas o los indicadores cifrados, considerando, o bien que las fuentes históricas no se prestaban a dichos usos, o bien que era más importante entender de forma cualitativa los procesos implicados insistiendo sobre las dinámicas o los procesos en funcionamiento.

Desde los años 1990, un análisis de redes más formalizado ha aparecido de forma episódica, y desigual según las áreas lingüísticas, en otros trabajos históricos fundados, por el contrario, en observaciones sistemáticas a una escala macro: es lo que sucede con los análisis de citas en historia de las ciencias, el estudio del parentesco $\mathrm{y}$, en particular, de las alianzas entre varias generaciones en antropología histórica o los estudios de los interlocks, es decir las relaciones entre empresas creadas por los administradores compartidos, en historia económica. En todo caso el objetivo de una aproximación mediante redes no es tanto mostrar la agencia individual como describir las estructuras, las zonad e mayor o menor cohesión o las formas de atracción preferntes: se trata más bien de aproximaciones estructurales que examinan las interacciones entre actores para interrogarse sobre sus efectos sistémicos.

Sin embargo, estas últimas tentativas se mantienen relativamente aisladas, a menudo en el margen de la disciplina - y particularmente en los puntos de encuentro con la sociología - pero sin contacto con las aproximaciones más microhistóricas de las relaciones sociales quienes, por su parte, se mantienen activas. Si nuestro número especial ha sido precedido de numerosos textos que, a la vez, esbozaban un estado del arte $y$, en general, un programa del análisis de redes en 
REDES- Revista hispana para el análisis de redes sociales

Vol.21,\#1, Diciembre 2011

http://revista-redes.rediris.es

historia $^{2}$, es notorio que los esfuerzos siguen estando relativamente aislados unos de otros, sin un verdadero diálogo científico entre aquellos que los llevan a cabo. Cada uno de estos artículos programáticos hablaba desde una postura un poco diferente $y$, si bien han dado lugar a numerosas investigaciones, en general se ha tratado más de un fenómeno de moda que de una verdadera aplicación de las orientaciones propuestas. Sin embargo, después de 30 años de una integración de la categoría de las redes al campo de la historia, de los cuestionamientos con los que está asociada y de las metodologías que implica, emerge un verdadero "savoirfaire" del historiador tal y como lo muestran las investigaciones que movilizan efectivamente los análisis de redes fomales llevados a cabo en el marco de las tesis leídas en los últimos años. Precisamente, y las investigaciones de la mayoría de los autores que contribuyen a este número lo atestiguan, se llega al análisis de redes, o bien durante la tesis, o bien inmediatamente después para revisitar con otro método, más sistemático, un tema de investigación bien conocido de un punto de vista cualitativo. Las formas de estructuración de la discusión sobre los usos de las redes en historia parecen también emerger a escala europea y a menudo, y no es por casualidad, han sido lanzadas y dinamizadas por doctorandos ${ }^{3}$.

\section{Objetivos y resultados de este número}

Si bien están emergiendo nuevas prácticas, lo hacen siempre en orden disperso, como lo muestra este número. Los propios coordinadores del número nunca fueron unánimes en sus apreciaciones sobre los textos recibidos, sus debates muestran la movilización permanente y diversa de una misma herramienta, el análisis de redes sociales, donde se entrecruzan y confrontan análisis cualitativos y aproximaciones más formalizadas y estructurales. Esta heterogeneidad se encuentra inevitablemente también entre los autores ${ }^{4}$ de los textos de este número: prácticamente no se citan entre ellos y, sobretodo, las referencias que movilizan

\footnotetext{
2 Se pueden mencionar en particular Erickson, 1997, Castellano y Dedieu, 1998, Wetherell, 1998, Bertrand, 1999, Gould, 2003, Lipp, 2003, Lemercier, 2005, 2012. Se encontrarán numerosas référencias a dichos trabajos en el principio de esta introducción.

3 Véase en concreto la "Network Analysis and History Conference", organizada en 2010 en la Universidad de Losana, Suiza de la cual vienen varios de los textos de este número (<http://www3.unil.ch/wpmu/reseaux2010/conference/program/>), la pagina web "Historical Network Research" (<https://sites.google.com/site/historicalnetworkresearch/>), la lista de difusión "The Networks Network" (<http://groups.google.com/group/the-networks-network >) y el próximo congreso previsto para 2012 en Southampton "The Connected Past" (<http://connectedpast.soton.ac.uk/>).

${ }^{4}$ Es evidente que la lengua sigue siendo una barrera lo que pone de relieve el papel de una revista como REDES. Hay que agradecer los esfuerzos de Ainhoa de Federico para coordinar las traducciones de los textos de este numero y de traducir ella misma una buena parte. Las traducciones de los textos han sido apoyados por el Fonds des Publications de l'Université de Lausanne, los laboratorios FRAMESPA y CERS-LISST de la Université de Toulouse II y Sciences Po Paris.
} 
REDES- Revista hispana para el análisis de redes sociales

Vol.21,\#1, Diciembre 2011

http://revista-redes.rediris.es

son también muy heterogéneas, en particular dependiendo de la lengua de los autores, pero también del tema de investigación sustantivo que estudian. De este modo, las discusiones históricas sobre las redes se refieren a autores, y en particular a teorías, muy variadas según el periodo estudiado o la subdisciplina de que se trate - historia de la familia y de las alianzas, de la empresa y de los consejos de administración, de las ciencias o las letras y de la correspondencia o las citas... Sucede lo mismo en función de las otras ciencias sociales frecuentadas o de los métodos utilizados. Estos últimos son más variados, yendo de colaboraciones directas con matemáticos o físicos a un rechazo total de la formalización pasando por toda la paleta de herramientas desarrolladas para la sociologíaa, que de todos modos hay que adaptar para datos diferentes.

De este modo, si el análisis de redes y ha demostrado su interés en algunas áreas específicas, el diálogo entre los que lo practican es escaso. Nuestro deséo, mostrando las relaciones y las differencias que existen entre textos provenientes de países y sub-disciplinas variadas de la disciplina, es promover dicho diálogo. Los textos publicados aquí revisitan algunos temas ya clásicos dentro del análisis de redes sociales, al menos en otras ciencias sociales: si el tema de las migraciones no es abordado, varios textos evocan las relaciones de parentesco (artículos de Padgett $y$, en parte, de Rosé y de Artola), las relaciones interpersonales subyacentes a las jerarquías entre élites (Padgett, Imizcoz et Arroyo, Rosé, Artola, Düring et al.), los interlocks (Ginalski), la circulación del saber (Widmer y Sigrist), las movilizaciones sociales y políticas (Rebmann, Düring et al.). Sin embargo, y este es uno de los puntos de interés de este número, nunca lo hacen unicamente copiando las maneras de hacer adoptadas por los sociólogos o los antropólogos en áreas de investigación similares, o aplicando paso a paso los manuales de análisis de redes sociales, calculando un indicador tras otro... Por el contrario, muestran una gran inventividad en materia de construcción de las fuentes, de las representaciones gráficas, de los cálculos de indicadores y/o de las interpretaciones para proponer una aproximación propiamente histórica de sus objetos de estudio.

Hemos efectuado una selección entre el gran número de artículos recibidos esperando remediar en parte la debilidad del debate sobre el análisis de redes sociales en historia. En particular, invitamos a los autores a explicitar, más allá de la presentación de un estudio de caso, sus elecciones teóricas y metodológicas y a esforzarse por tender hacia aportes generalizables a partir de sus experiencias de investigación. Les pedimos que explicasen su tratamiento del anacronismo (¿se pueden describir las relaciones sociales del pasado con las palabras utilizadas para hablar de las nuestras?), que tratasen la cuestión de la temporalidad y por lo tanto 
REDES- Revista hispana para el análisis de redes sociales

Vol.21,\#1, Diciembre 2011

http://revista-redes.rediris.es

de las evoluciones o los cambios - ahí también una fuente de problemas de colecta o de representación de datos específicos de historia a la vez que una promesa de análisis más finos.

Finalmente les incitamos a explicitar su relación con las fuentes históricas escritas o figuradas, raras veces disponibles de forma sistemática y que, al contrario que los cuestionarios o las entrevistas, representan más bien, en general, huellas de interacciones que un discurso sobre dichas interacciones: ¿dan problemas particulares - o presentan un interés particular - par un estudio en términos de redes? Cualquier fuente histórica es parcial, no sistemática. Las contribuciones reunidas aquí muestran a la vez las diferencias entre los periodos antiguos y medievales de los que no se dispone mas que de fuentes fragmentadas e indirectas únicamentes sobre algunos miembros de la élite política y otras épocas para las que, al menos en ciertos aspectos de la vida social, los tratamientos sistemáticos se vuelven posibles, aunque sigan estando centrados en las élites, en el sentido de aquellas personas que saben escribir (como es el caso de la Toscana del siglo XV o la España del XVII). Si el mundo organizacional de las empresas o de las comisiones políticas del siglo XX permite más fácilmente constituir grandes bases de datos, el problema es entonces escoger la parte de la realidad a observar, en particular en términos de cortes temporales, otros artículos muestran también que sería reductor reservar el análisis de grandes redes a los periodos más recientes. Así pues, al definir precisamente la relación a observar y la confianza que se puede tener en los trabajos preexistentes de historiadores, es posible trazar una genealogía entre cientos de botanistas del siglo XVIII, mientras que la representación de las relaciones que permiten salvar a los judíos en el siglo XX debe manejarse con los sesgos y los silencios de las fuentes.

En nuestra selección final de los artículos hemos privilegiado aquéllos que proponen análisis formales de redes sociales incluida su representación gráfica. No se trata en absoluto de una elección a favor de aproximaciones macro, estructurales o técnicamente pesadas: muchas de las redes presentadas aquí son muy pequeñas y/o egocentradas y todas son estudiadas en el marco de investigaciones más amplias para las que la cuantificación es una de las herramientas utilizadas puntualmente. Sin embargo, hemos querido evitar publicar estudios, todavía frecuentes en historia que únicamente utilizan el concepto de red como una metáfora vaga, y hemos privilegiado la sistematicidad de las aproximaciones y la inventividad metodológica. No se trata sin embargo de un amor de la técnica por la técnica. Pedimos a cada autor en particular que explicitase de que manera aportaba a su argumentación el uso de una aproximación formalizada de las redes: 
REDES- Revista hispana para el análisis de redes sociales

Vol.21,\#1, Diciembre 2011

http://revista-redes.rediris.es

¿producen conocimientos e interpretaciones que no serían accesibles con una aproximación intuitiva o cualitativa sin acceso a la informática, a los grafos o a los indicadores numéricos?

Nos parece que han producido respuestas convincentes porque su uso de la formalización está al servicio de su argumentación histórica, lo que les ha permitido precisamente ser innovatigos. De este modo, otros investigadores se podran inspirar, en historia, pero sin duda también en otras ciencias sociales, de las representaciones gráficas heurísticas propuestas por Düring et al., de la cómo se tiene en cuenta el tiempo en la de Rosé y la de Sigrist y Widmer y de los cambios de escala flexibles permitidos por la de Ginalski ; o aún, por el lado de la construcción de datos, de cómo se tienen en cuenta, no sólo las relaciones de correspondencia, sino también las relaciones de citación en la corresondencia de Imizcoz y Arroyo, de la articulación entre los estudios de trayectorias y representaciones de redes puestos en práctica por Artola, finalmente, del punto de vista de los indicadores, el uso sutil de Rebmann del concepto de homofilia, en un contexto institucional inhabitual. La mayor parte del tiempo estas formalizaciones conservan un estatus exploratorio en los artículos: muestran una estructura compleja, a menudo reconstituida a partir de un gran conjunto de fuentes, que no se podría percibir a partir de una simple lectura. Sin embargo pocos artículos tratan de utilizar el análisis de redes para poner a prueba hipótesis: esta es una diferencia real entre la historia y la sociología, bien presentada por Padgett en su texto introductorio. 
REDES- Revista hispana para el análisis de redes sociales

Vol.21,\#1, Diciembre 2011

http://revista-redes.rediris.es

\section{Problemas y perspectivas: ¿qué hacen las redes?}

No pretendemos que, a pesar de algunos rasgos comunes, los artículos reunidos aquí hayan salido del mismo molde, ni siquiera que compartan una aproximación precisa de las redes. Es cierto que todos ellos comparten la convicción de que los lazos personales, las redes de relaciones individuales, dan nacimiento a formas de organización sociales que van más allá de los marcos institucionales, los grupos formales, las clases socio-económicas y a dinámicas diferentes, y que es esencial tenerlas en cuenta para comprender una formación social y, sobretodo, su evolución y su transformación. Dicho esto, el análisis sistemático de las grandes redes y el estudio más cualitativo de las pequeñas redes personales siguen apareciendo como polos bastantes diferenciados y que no otorgan el mismo estatus al concepto de red en sus demostraciones.

De este modo, en una parte de los estudios, se pone el acento en las aproximaciones estructurales en términos de redes completas, que tratan de explicitar las formas de organización de un grupo o de un segmento de la sociedad y compararlas en el tiempo, pero también respecto a las formas esperadas en función de diferentes hipótesis previas (Padgett, Ginalski, Rebmann). Otros estudios privilegian aproximaciones más estratégicas; de forma más narrativa, se focalizan en los espacios de acción, las elecciones y las estrategias de los individuos y de los grupos (Rosé, Imizcoz et Arroyo, Artola) ${ }^{5}$. La temporalidad, en particular, no es la misma en ambos casos; el estatus de las relaciones tampoco, dado que son más bien vistas, en el primer caso, como canales potenciales que pueden ser movilizados para objetivos diversificados, mientras que en el segundo caso, ésta movilización efectiva - el agua que circula, o no, en esos canales - es la que se trata de describir ${ }^{6}$. En otros términos, sigue siendo la doble movilización de la herramienta de las redes la que está en juego aquí.

Sin embargo, como lo subraya la confrontación de los textos, estas dos aproximaciones no son, evidentemente, exclusivas. Este número monográfico ofrece pistas sobre convergencias posibles. La investigación de A. Artola sobre las

\footnotetext{
${ }^{5}$ Un artículo de Martin Stuber y Lothar Krempel presentado en Lausanne en 2010 y recibido demasiado tarde para poder figurar en este numéro, "The scholarly networks of Albrecht von Haller and the Economic Society - a multi-level network analysis", muestra particularmente esta dicotomía presentando primero las cadenas relacionales efectivamente utilizadas para lanzar experiencias agronómicas y después de las redes que existen más como potencialidades, basadas en la correspondencia regular o las afiliaciones institucionales. Sobre el concepto de cadena relacional, que podría ser particularmente heurístico en historia, véase Grossetti et al., 2011.
} 
REDES- Revista hispana para el análisis de redes sociales

Vol.21,\#1, Diciembre 2011

http://revista-redes.rediris.es

trayectorias profesionales de los miembros del clero español examina a la vez las carreras y las estrategias individuales y las estructuras recurrentes de reclutamiento de dicho personal eclesiástico. El estudio de las trayectorias de poder de Odon de Cluny por Isabelle Rosé se situa igualmente en la intersección entre estructuras sociales y estrategias individuales. En el estudio de la domus augusta del artículo de Düring et al., los autores subrayan de forma particularmente explicita la interacción entre las estructuras de parentesco y estrategias conscientes de los actores, que presentan en términos de « espacios de acción » que abren a las posibilidades a « emprendedores de redes ». Así, los estudios históricos, con una mirada atenta a la cronologíaa y a la dinámica de las relaciones sociales y un acceso a las fuentes que permiten identificar las huellas de interacciones reales y no solo potenciales, tienen algo que decir sobre las modalidades de interacción entre estructuras sociales y la agencia individual. Es precisamente esta interacción la que pone en evidencia el estudio reciente de las redes mercantiles a escala imperial hispánica. El recurso a la confianza y la necesidad de acceso a la información, dos de los fundamentos sobre los que se apoyan las redes estudiadas orientando las operaciones comerciales de sus miembros y conjugándose con las estructuras sociales pero también con las instituciones mercantiles mismas - para dar a entender, en toda su complejidad, las modalidades de funcionamiento de dicho comercio transatlántico y las operaciones puntuales implicadas. ${ }^{7}$

Pero no todos los autores son tan explicitos en lo que respecta a su posición relativa a este tema, como, de forma más general, en lo que concierne el estatus causal que asignan a las redes. A menudo, en una situación documental llena de lagunas, el análisis de redes «visibles » parece la base de una estrategia de interpretación adaptada a dichas lagunas: para simplificar, si no sabemos exactamente lo que hacen los actores, podemos al menos obervar ciertas formas y tratar de construir, por lo menos, una interpretación del sentido de las relaciones que las delinéan. Esta estrategia puede ser a pesar de todo arriesgada si se mantiene una definición vaga del estatus de las redes como instrumento conceptual en la interpretación histórica $y$, en particular, de las consecuencias que se pueden derivar de la reconstrucción de una red específica. En particular, cuando nace de la crítica de aproximaciones demasiado deterministas, el análisis de redes presenta el riesgo de introducir una nuevo determinismo incontrolado, si produce una fascinación por la forma de la

6 Sobre estas différencias entre las redes como « canales y las redes como historias véase White 2011.

${ }^{7}$ Véase especialmente a este respecto E. Van Young, 2011. 
REDES- Revista hispana para el análisis de redes sociales

Vol.21,\#1, Diciembre 2011

http://revista-redes.rediris.es

estructura descubierta olvidando qué relaciones precisas implica y qué sentido podían tener para los actores.

El ejemplo de los interlocks y más ampliamente de las relaciones entre organizaciones establecidas a partir de miembros compartidos es clásica: la interpretación de las formas obtenidas se basa en una serie de hipótesis, cierto, plausibles, pero pocas veces discutidas en detalle: la existencia de relaciones personales entre los diferentes miembros de consejos de administración permite un intercambio de información, establece una confianza o facilita la cooperación. Aquí, Ginalski y Rebmann se toman el tiempo de explicitar, a propósito de los mecanismos que les interesan, lo que tal o tal forma de red podría indicar. Sigue siendo cierto que no estamos frente a un estudio directo de los procesos: a menudo es difícil demostrar cómo actúa la red, la acción - lo que la red "hace" - se mantiene por lo tanto implícito o hipotético. Como lo señalan Düring et al., la acción de la red es tanto más difícil a demostrar cuando las fuentes son menos sistemáticas.

Si bien no siempre es posible resolver este problema, hay que mantenerlo en mente: nunca es equivalente observar la movilización de relaciones con un objetivo preciso o mostrar las relaciones potenciales, que serán o no activadas, tanto si forman parte del parentesco, de la co-participación a una organización o la mención en una correspondencia. En el segundo caso, no hay que sobreestimar el estatus explicativo de una estructura de relaciones, tanto si se trata de elucidar las causas de un éxito individual, una cooperación eficaz, un intercambio privilegiado de informaciones o aún la existencia misma de un grupo social significativo - y ello tanto más que Is fuentes no permiten observar a menudo mas que uno o dos tipos de relaciones sociales entre todas las que pueden movlizar los individuos.

En resumen, mostrar estructuras similares entre las redes no dispensa de interesarse a la sustancia misma de las relaciones (Bidart et al., 2009), tanto más que este interés implica la crítica de las fuentes que se sitúan en el corazón del oficio de historiador/a. El caso de la « familia » y del parentesco, que finalmente no están en el centro, al menos de forma sustantiva, de ninguno de los artículos de este número, es particularmente ejemplar en la materia. Estas se evocan a menudo, con cierta facilidad de razonamiento, para explicar colaboraciones privilegiadas entre actores sociales. Sin embargo el parentesco es la base de una relación estructura, en parte involuntaria, que no implica automáticamente relaciones estrechas, ni tan solo un conocimiento recíproco - en el caso de parientes alejados, o que residen en regiones diferentes, por ejemplo. Se trata 
REDES- Revista hispana para el análisis de redes sociales

Vol.21,\#1, Diciembre 2011

http://revista-redes.rediris.es

entonces de lazos muy diferentes a los establecidos, por ejemplo, en el marco de una relación epistolar que es claramente objeto de una elección voluntaria fundada en una cierta afinidad. Pierre Bourdieu ya había subrayado la diversidad de los usos posibles del parentesco, distinguiendo el parentesco "oficial" y el parentesco "usual" en sus investigaciones sobre la sociedad kabil. En este número, Imizcoz y Arroyo muestran que un análisis de la correspondencia permite describir ciertas modalidades de activación de las relaciones de parentesco. Aquí de nuevo, la estructura y la agencia se combinan y esclarecen de forma original lo que « hace la red » y evitar toda sobredeterminación o sobreinterpretación. Otras distinciones podrían ser introducidas en el análisis de las relaciones de parentesco, en particular entre el parentesco patrilineal (a menudo privilegiado en las investigaciones formalizadas, dado que puede ser seguida, en la mayoría de las sociedades, a partir del apellido) y parentesco matrilineal, a la vez más difícil de reconstituir y con consecuencias sociales eventualmente diferentes.

El parentesco espiritual, que no hace mas que una aparición puntual en este número exacerba el problema. Si la relación establecida a partir del bautizo con uno o varios padrinos y madrinas es un rasgo muy generalizado de las sociedades católicas y protestantes, así como tantas otras organizaciones sociales, el contenido concreto de esta relación puede variar de forma sustantiva, por ejemplo, hoy en día esta relación tiende a ser socialmente más « horizontal » que en el pasado. Los estudios sobre este tema que comienzan a utilizar el análisis de redes muestran una via posible: utilizan a la vez reconstituciones sistemáticas que permiten proponer hipótesis razonables sobre los usos sociales implicitos ** no admitidos de la institución, por ejemplo sobre sus aspectos de patronazgo implícito o su papel de canalización de movilizaciones políticas y de fuentes complementarias numerosas utilizadas de forma más cualitativa para comprender mejor lo que los actores dicen de dichos lazos (Alfani 2006, Guzzi-Heeb 2009).

Los trabajos más finos sobre la familia muestran un último problema que se mantiene demasiado implícito en los artículos de este número como en la mayoría de los estudios de análisis de redes. A veces parece evidente que las unidades cuyas relaciones se estudian son todas de igual naturaleza: individuos o bien colectivos más o menos formalizados. Sin embargo, si hablamos de una alianza, ¿acaso no crea relaciones más allá de las personas que contraen matrimonio? ¿Únicamente entre personas cercanas? ¿entre otras personas de entre sus parientes? ¿No sería más justo considerarla como una alianza entre grupos? ¿Y no sería importante evitar reducir dichos grupos, por comodidad, más que a un parentesco patrilineal (es decir patronímico) para definir con mayor precisión quién 
REDES- Revista hispana para el análisis de redes sociales

Vol.21,\#1, Diciembre 2011

http://revista-redes.rediris.es

se liga? Varios estudios sugieren así que la realción esencial establecida por el parentesco espiritual no es entre dos individuos sino entre dos familias o dos grupos de parentesco. Los estudios de las redes « bimodales », como los interlocks, esbozan con demasiada frecuencia cuestiones del mismo tipo: los lazos que se crean en ellos siguen una lógica exclusiva o bien de elección de individuos por individuos (seleccionando por ejemplo los administradores de su familia) o bien de organizacions con organizaciones (empresas de un mismo sector que preparan, por ejemplo, una fusión), sin embargo, ¿no es más plausible que ambos niveles interactúen? Los artículos de Ginalski y Rebmann lo dejan presentir, pero se podría ir más lejos en la explicitación de dicha cuestión.

Siguen quedando, por lo tanto, varias cuestiones que merecen ser profundizadas por investigaciones posteriores: esto nos parece un signo de fecundidad del concepto de red para las invstigaciones históricas y de sus potencialidades en la perspectiva de una mejor comprensión de dinámicas sociales complejas.

\section{Bibliografía}

Bertrand, Michel (1999). «De la familia a la red de sociabilidad », Revista Mexicana de Sociología, vol. 61, № 2, avril-juin, pp. 107-135.

Bidart, Claire; Johanne Charbonneau \& Michel Grossetti (editores) (2009). Monográfico "Las relaciones: la sustancia de las redes sociales." Revista REDES. Revista hispana para el análisis de redes sociales. Vol 16 (Junio).

Castellano, Juan Luis \& Jean-Pierre Dedieu (dir.) (1998). Réseaux, familles et pouvoirs dans le monde ibérique à la fin de l'Ancien Régime, Paris: CNRS Éditions.

Erickson, Bonnie H. (1997). Social Networks and History. "A Review Essay", Historical Methods, vol. 30, issue 3 (Summer), 149-157.

Gould, Roger V. (2003). "Uses of network tools in comparative historical research", in: James Mahoney and Dietrich Rueschemeyer, eds., Comparative historical analysis in the social sciences. Cambridge: Cambridge University Press, pp. 241269.

Grossetti, Michel, Jean-François Barthe \& Nathalie Chauvac (2011). "Studying Relational Chains from Narrative Material", Bulletin de méthodologie sociologique, no 110 , pp. 11-25. 
REDES- Revista hispana para el análisis de redes sociales

Vol.21,\#1, Diciembre 2011

http://revista-redes.rediris.es

Lemercier, Claire (2005). «Analyse de réseaux et histoire », Revue d'histoire moderne et contemporaine, vol. 52, $n^{\circ} 2$ (Avril-Juin).

Lipp, Carola (2003). "Struktur, Interaktion, räumliche Muster. Netzwerkanalyse als analytische Methode und Darstellungsmittel sozialer Komplexität", in: Silke Göttsch, and Christel Köhle-Hezinger, eds., Komplexe Welt. Kulturelle Ordnungssysteme als Orientierung, Münster, pp. 49-63.

Van Young, Eric (2011). « Social Networks: A Final Comment », in: Nikolaus Böttcher, Bernd Hausberger, Antonio Ibarra (Coords), Redes y negocios globales en el mundo ibérico, siglos 16-18, México: El Colegio de México, pp. 289-309.

Wetherell, Charles (1998). « Historical social network analysis », International Review of Social History, 43, supplement 6, p. 125-144. 